\title{
Future Type Ia Supernova Data as Tests of Dark Energy from Modified Friedmann Equations
}

\author{
Yun Wang, Katherine Freese, ${ }^{2}$ Paolo Gondolo, ${ }^{3}$ and Matthew Lewis ${ }^{4}$
}

\begin{abstract}
In the Cardassian model, dark energy density arises from modifications to the Friedmann equation, which becomes $H^{2}=g\left(\rho_{M}\right)$, where $g\left(\rho_{M}\right)$ is a new function of the energy density. The universe is flat, matter dominated, and accelerating. The distance redshift relation predictions of generalized Cardassian models can be very different from generic quintessence models, and can be differentiated with data from upcoming pencil beam surveys of Type Ia Supernovae such as SNAP. We have found the interesting result that, once $\Omega_{m}$ is known to $10 \%$ accuracy, SNAP will be able to determine the sign of the time dependence of the dark energy density. Knowledge of this sign (which is related to the weak energy condition) will provide a first discrimination between various cosmological models that fit the current observational data (cosmological constant, quintessence, Cardassian expansion). Further, we have performed Monte Carlo simulations to illustrate how well one can reproduce the form of the dark energy density with SNAP.

To be concrete we study a class of two parameter $(n, q)$ generalized Cardassian models that includes the original Cardassian model (parametrized by $n$ only) as a special case. Examples are given of MP Cardassian models that fit current supernovae and CMB data, and prospects for differentiating between MP Cardassian and other models in future data are discussed. We also note that some Cardassian models can satisfy the weak energy condition $w>-1$ even with a dark energy component that has an effective equation of state $w_{X}<-1$.
\end{abstract}

\footnotetext{
${ }^{1}$ Department of Physics \& Astronomy, University of Oklahoma, Norman, OK 73019

${ }^{2}$ Michigan Center for Theoretical Physics, Physics Department, University of Michigan, Ann Arbor, MI 48109, USA, and Kavli Institute for Theoretical Physics, University of California, Santa Barbara, CA 93106

${ }^{3}$ Department of Physics, Case Western Reserve University, 10900 Euclid Ave, Cleveland, OH 44106-7079

${ }^{4}$ Michigan Center for Theoretical Physics, Physics Department, University of Michigan, Ann Arbor, MI 48109, USA
} 


\section{Introduction}

Recent observations of Type Ia Supernovae (Riess et al. 1998; Perlmutter et al. 1999) as well as concordance with other observations (including the microwave background and galaxy power spectra) indicate that the universe is accelerating. Many authors have explored a cosmological constant, a decaying vacuum energy (Freese et al. 1987; Peebles \& Ratra 1988; Frieman et al 1995), quintessence (Wang \& Steinhardt 1998; Caldwell, Dave, \& Steinhardt 1998; Huey et al. 1999), and gravitational leakage into extra dimensions (Deffayet 2001) as possible explanations for such an acceleration. Recently Freese \& Lewis (2002) proposed Cardassian expansion as an explanation for acceleration which invokes no vacuum energy whatsoever. ${ }^{5}$ In this model the universe is flat and accelerating, and yet consists only of matter and radiation.

In Cardassian models, the Friedmann equation is modified from $H^{2}=8 \pi \rho /\left(3 m_{p l}^{2}\right)$ to

$$
H^{2}=g\left(\rho_{M}\right)
$$

where $g\left(\rho_{M}\right)$ is a different function of the energy density, $\rho_{M}$ contains only matter and radiation (no vacuum), $H=\dot{a} / a$ is the Hubble constant (as a function of time), and $a$ is the scale factor of the universe. Models with gravitational leakage into extra dimensions also give a modified Friedmann equation that can be cast into the form of Eq. (1), but with a vacuum component.

The function $g\left(\rho_{M}\right)$ returns to the usual $8 \pi \rho_{M} /\left(3 m_{p l}^{2}\right)$ during the early history of the universe, but takes a different form that drives an accelerated expansion after a redshift $z \sim 1$. Such modifications to the Friedmann equation may arise, e.g., as a consequence of our observable universe living as a 3-dimensional brane in a higher dimensional universe (Chung \& Freese 2000). Alternatively, such a Friedmann equation may arise if there is dark matter with self-interactions characterized by negative pressure (Gondolo \& Freese 2002).

We wish to study the detectability of the altered Friedmann equations by upcoming observations of Type Ia Supernova such as SNAP. As discussed in Freese (2002), the redshift distance relationship predictions for generalized Cardassian models can be quite different from generalized quintessence models. It is the goal of this paper to see how well we can reproduce the correct form of this dark energy density in upcoming experiments.

For concreteness, we investigate a particular version of generalized Cardassian cosmology. However, our results are intended to generalize to any Cardassian cosmology, i.e., to

\footnotetext{
${ }^{5}$ The name Cardassian refers to a humanoid race in Star Trek whose goal is to accelerate expansion of their evil empire. This race looks alien to us and yet is made entirely of matter.
} 
any function $g\left(\rho_{M}\right)$. The particular model we study is meant to illustrate that, generically, modified Friedmann equations can lead to specific detectable predictions in experiments like SNAP.

In this paper we study the following generalization of the original Cardassian model:

$$
H^{2}=\frac{8 \pi G \rho_{M}}{3}\left[1+\left(\frac{\rho_{\text {Card }}}{\rho_{M}}\right)^{q(1-n)}\right]^{1 / q} .
$$

We call this model "Modified Polytropic Cardassian" (MP Cardassian) ${ }^{6}$ (Gondolo \& Freese 2002), where $G=1 / m_{p l}^{2}$ is Newton's universal gravitation constant, $\rho_{\text {Card }}$ is a characteristic constant energy density, and where we take $n<2 / 3$ and $q>0$. The original power law Cardassian model corresponds to $q=1$.

For comparison, we remind the reader of the original power law Cardassian model that was proposed in Freese \& Lewis (2002), which had the following specific form of $g\left(\rho_{M}\right)$ :

$$
H^{2}=\frac{8 \pi}{3 m_{p l}^{2}} \rho_{M}+B \rho_{M}^{n} \quad \text { with } \quad n<2 / 3
$$

This is equivalent to writing

$$
H^{2}=\frac{8 \pi G}{3} \rho_{M}\left[1+\left(\frac{\rho_{\text {Card }}}{\rho_{M}}\right)^{1-n}\right],
$$

The first term inside the bracket of Eq.(2) and Eq.(4) dominates initially, so that ordinary Friedmann Robertson Walker (FRW) behavior takes place throughout the early universe. At a redshift $z_{\text {Card }} \sim 1$, the two terms inside the bracket become equal, and henceforth the second term dominates. Once the second term dominates, it drives the universe to accelerate. The energy density at which the two terms become equal is $\rho_{\text {Card }}=\rho_{0}\left(1+z_{\text {Card }}\right)^{3}$, where $\rho_{0}$ is the matter density today. The MP Cardassian model of Eq.(2) depends on three parameters: the numbers $n$ and $q$ and the density $\rho_{\text {Card }}$. The latter can be traded for the observed matter mass density $\Omega_{m}^{\text {obs }}$ (see Eq. [12] below).

The original power law Cardassian model gave the same distance-redshift relation as a quintessence model with constant equation of state parameter $w_{q}=n-1$. Generalized Cardassian models, on the other hand, give predictions for the distance-redshift relation that can be very different from generic quintessence models. For example, some Cardassian

\footnotetext{
${ }^{6}$ The name "Modified Polytropic" arises in the context of treating this model as a fluid; then the relationship between energy density and pressure is roughly polytropic (see Gondolo \& Freese (2002)).
} 
models can satisfy the weak energy condition $w>-1$ even with a dark energy component that has an effective equation of state $w_{X}=p_{X} / \rho_{X}<-1$. Note that an effective $w_{X}<-1$ is consistent with recent CMB and large scale structure data (Schuecker et al. 2002; Melchiorri et al. 2002). In this paper we explore these differences and their testability for the MP Cardassian model.

The Cardassian model also has the attractive feature that matter alone is sufficient to provide a flat geometry. Because of the extra term on the right hand side of the Friedmann equation, the critical mass density necessary to have a flat universe can be modified, e.g. to 0.3 of the usual value. Hence the matter mass density can have exactly this new critical value and satisfy all the observational constraints such as given by the baryon cluster fraction and the galaxy power spectrum.

\section{General Notation:}

In a flat universe, the total energy density of the universe can be written as

$$
\rho_{\text {total }}(z)=\rho_{c, \text { old }}\left[\Omega_{m}^{o b s}(1+z)^{3}+\Omega_{X} f_{X}(z)\right] .
$$

Here, $\Omega_{m}^{o b s}$ is the observed matter density of the universe; we will take $\Omega_{m}^{o b s}=0.3$ as our fiducial value. The critical density of the universe (for the ordinary Friedmann equation) is $\rho_{c \text {,old }}=2 \times 10^{-29} h_{0}^{2} \mathrm{gm} / \mathrm{cm}^{3}$, where $h_{0}$ is the Hubble constant today in units of 100 $\mathrm{km} / \mathrm{s} / \mathrm{Mpc}$. We take

$$
\Omega_{X}=1-\Omega_{m}^{o b s}
$$

(for a flat universe with total energy density $\Omega_{t o t}=1$ ), and $f_{X}(z=0)=1$. The subscript $X$ refers to any component of the universe that provides an additional term in Einstein's equation; generically it is called "dark energy", but in the Cardassian case it is an additional matter term. The dark energy density is

$$
\rho_{X}(z)=\rho_{X}(0) f_{X}(z)=\rho_{c, \text { old }} \Omega_{X} f_{X}(z) .
$$

If the dark energy density corresponds to a cosmological constant, then one finds that $f_{X}(z)=$ 1 at all redshifts $z$.

Note that for generalized Cardassian models, both terms in Eq. (5) come from matter;

$$
\Omega_{m}^{t o t}=\Omega_{m}^{o b s}+\Omega_{X}=1 .
$$

For the MP generalized Cardassian models of Eq. (2), the dimensionless dark energy density $f_{X}(z)$ is given by

$$
f_{X}(z)=\frac{\rho_{X}(z)}{\rho_{X}(0)}=\frac{\Omega_{m}^{o b s}(1+z)^{3}}{1-\Omega_{m}^{o b s}}\left\{\left[1+\frac{\left(\Omega_{m}^{o b s}\right)^{-q}-1}{(1+z)^{3(1-n) q}}\right]^{1 / q}-1\right\}
$$


For fixed $\Omega_{m}^{o b s}$, it depends on the two dimensionless parameters $n$ and $q$.

In Cardassian models, the observed matter mass density fraction today is given by the ratio of the critical mass density of the Cardassian universe, $\rho_{c \text {,Card }}=\rho_{0}$, and that of the standard universe, $\rho_{c \text {,old }} \equiv 3 H_{0}^{2} /(8 \pi G)$. So the observed matter mass fraction today in the MP Cardassian model is (Freese \& Lewis 2002)

$$
\Omega_{m}^{o b s}=\frac{\rho_{0}}{\rho_{c, \text { old }}}=\frac{1}{\left[1+\left(1+z_{\text {Card }}\right)^{3 q(1-n)}\right]^{1 / q}} .
$$

Inversely, we can express $z_{\text {Card }}$, and $\rho_{\text {Card }}$, in terms of $\Omega_{m}^{o b s}$ as

$$
1+z_{\text {Card }}=\left[\left(\frac{1}{\Omega_{m}^{\text {obs }}}\right)^{q}-1\right]^{\frac{1}{3 q(1-n)}}
$$

and

$$
\rho_{\text {Card }}=\rho_{c, \text { old }} \Omega_{m}^{o b s}\left[\left(\frac{1}{\Omega_{m}^{o b s}}\right)^{q}-1\right]^{\frac{1}{q(1-n)}} .
$$

Outline: First, we will compare the Modified Polytropic Cardassian model of Eq.(2) with existing data from supernovae and cosmic microwave background data.

Next, we explore how plausible future SNe Ia data can be optimally used to constrain dark energy models, and whether generalized Cardassian models can be differentiated from generic models of quintessence and models with a cosmological constant.

Most of this work was performed in September 2002 when all the authors were at the Kavli Institute for Theoretical Physics in Santa Barbara. Subsequent dispersal of all the authors to different parts of the country caused the conclusion of the paper to take a long time.

\section{Comparison of MP Cardassian with Current Data}

In this section, we compare the Modified Polytropic Cardassian Model of Eq. (2) with current supernovae and cosmic microwave background (CMB) data. We will see that the existing data can be well fit for several choices of the parameters $n$ and $q$.

In a smooth Friedmann-Robertson-Walker (FRW) universe, the metric is given by $d s^{2}=$ $d t^{2}-a^{2}(t)\left[d r^{2} /\left(1-k r^{2}\right)+r^{2}\left(d \theta^{2}+\sin ^{2} \theta d \phi^{2}\right)\right]$, where $a(t)$ is the cosmic scale factor, and $k$ is the global curvature parameter. The comoving distance $r$ is given by (Weinberg 1972)

$$
r(z)=c H_{0}^{-1} \frac{S(\kappa \Gamma)}{\kappa}, \quad \kappa \equiv\left|\Omega_{k}\right|^{1 / 2}
$$




$$
\begin{gathered}
\Gamma\left(z ; \Omega_{m}^{o b s}, \Omega_{X}, F\right)=\int_{0}^{z} d z^{\prime} \frac{1}{E\left(z^{\prime}\right)} \\
E\left(z^{\prime}\right) \equiv\left[\Omega_{m}^{o b s}\left(1+z^{\prime}\right)^{3}+\Omega_{X} f_{X}\left(z^{\prime}\right)+\Omega_{k}\left(1+z^{\prime}\right)^{2}\right]^{1 / 2}
\end{gathered}
$$

where $\Omega_{k}=1-\Omega_{m}^{o b s}-\Omega_{X}$, and

$$
\begin{aligned}
S(x) & =\sinh (x), & & \Omega_{k}>0 \\
& =x, & & \Omega_{k}=0 \\
& =\sin (x), & & \Omega_{k}<0 .
\end{aligned}
$$

The angular diameter distance is given by $d_{A}(z)=r(z) /(1+z)$, and the luminosity distance is given by $d_{L}(z)=(1+z) r(z)$.

The distance modulus for a standard candle at redshift $z$ is

$$
\mu_{p}(z) \equiv m-M=5 \log \left(\frac{d_{L}(z)}{\mathrm{Mpc}}\right)+25,
$$

where $m$ and $M$ are the apparent and absolute magnitudes of the standard candle, and $d_{L}(z)$ is its luminosity distance.

Type Ia supernovae (SNe Ia) are our best candidates for cosmological standard candles, because they can be calibrated to have small scatters in their peak luminosity (Phillips 1993; Riess, Press, \& Kirshner 1995).

Fig. 1 shows the measured distance modulus (actually the deviation of the distant modulus with respect to the expected values for an open universe with $\Omega_{m}^{o b s}=0.3$ and $\Omega_{\Lambda}=0$ ) for flux-averaged ${ }^{7}$ (Wang 2000b) SNe Ia data (Riess et al. 1998; Perlmutter et al. 1999) as a function of redshift. For comparison, superposed on the data points are the

\footnotetext{
${ }^{7}$ Here we briefly describe flux-averaging. Due to the inhomogeneous distribution of matter in our universe, the light from perfect standard candles (which all have exactly the same peak luminosity) at a given redshift $z$ will experience different amounts of bending (due to matter inhomogeneity along different lines of sight) before reaching the observer. Hence even perfect standard candles will be observed to have a non-Gaussian spread in peak luminosity due to gravitational lensing (Frieman 1997; Wambsganss et al. 1997; Holz \& Wald 1998; Metcalf \& Silk 1999; Wang 1999; Wang, Holz, \& Munshi 2002; Munshi \& Wang 2003). If this effect is not properly taken into account, the estimated cosmological parameters will be biased, i.e., the estimated mean of the parameters will deviate from the true value of the parameters. Fortunately, the total number of photons from all the standard candles at redshift $z$ should remain unchanged in the presence of gravitational lensing (which only redistributes the photons by bending the light from each standard candle); therefore the average peak luminosity of all the standard candles at $z$ should be the same as the peak luminosity of a standard candle at $z$ without gravitational lensing. This is the basic idea behind flux-averaging of type Ia supernova data. For details, see Wang (2000b).
} 
predictions for several familiar cosmological models (dotted curves, from top to bottom): $\left(\Omega_{m}^{o b s}, \Omega_{\Lambda}\right)=(0.3,0.7) ;(0.3,0)$; and $(1,0)$. In addition, three examples of modified polytropic Cardassian models from Eq. (2) are shown, all with $\Omega_{m}^{\text {obs }}=0.3$. The three models have parameters $n=0.2, q=1$ (solid curve); $n=0.2, q=2$ (short-dashed curve); and $n=0.2$, $q=3$ (long-dashed curve). Note that the solid curve is equivalent to a quintessence model with $w_{q}=-0.8$. Also shown in Fig.1 is a quintessence model with $w_{q}(z)=-1+0.5 z$ for comparison (dot-dashed line). All three generalized Cardassian models shown satisfy current constraints from SNe Ia. So we conclude that MP Cardassian models fit the existing supernovae data very well.

Fig. 2 shows the $(n, q)$ parameter space with constraints from current observational data from both supernovae and the cosmic microwave background (CMB), at a fixed value $\Omega_{m}=0.3$. The MP Cardassian model with parameters $(n, q)$ is compared with a fiducial $\Lambda \mathrm{CDM}$ (cold dark matter) model $\Omega_{m}=0.3, \Omega_{b}=0.05, \Omega_{\Lambda}=0.7$, and $h=0.65$. The constraints are derived by requiring that the MP Cardassian models agree with the fiducial $\Lambda \mathrm{CDM}$ model to within $1 \sigma$ of the measurement uncertainties of the Wilkinson Microwave Anisotropy Probe (WMAP) CMB data (Bennett et al. 2003) and the current SN Ia data [(Riess et al. 1998; Perlmutter et al. 1999), flux-averaged with $\Delta z=0.05$ (Wang 2000b)].

The region between the thick solid lines in Fig. 2 corresponds to $(n, q)$ values of the MP Cardassian models that satisfy the current SN Ia data [flux-averaged with $\Delta z=0.05$ ] within one sigma of the fiducial $\Lambda \mathrm{CDM}$ model, i.e., $\Delta \chi^{2}=\chi_{M P C}^{2}-\chi_{\Lambda \mathrm{CDM}}^{2}=1$. Note that the fiducial $\Lambda \mathrm{CDM}$ model $\left(\Omega_{m}=0.3, \Omega_{\Lambda}=0.7, h=0.65\right)$ corresponds to $n=0, q=1$. Complementary plots showing constraints from the power law Cardassian model with fixed $q=1$ and varying $n$ and $\Omega_{m}$ are given in Sen \& Sen (2002); Zhu \& Fujimoto (2002, 2003).

The dashed line in Fig. 2 indicates the constraints from WMAP CMB data. When the cosmological parameters are varied, the shift in the whole CMB angular spectrum is determined by the shift parameter (Bond, Efstathiou, \& Tegmark 1997; Melchiorri et al. 2002; Ödman at el. 2002)

$$
\mathcal{R}=\sqrt{\Omega_{m}} H_{0} r\left(z_{\text {dec }}\right)
$$

where $r\left(z_{\text {dec }}\right)$ denotes the comoving distance to the decoupling surface in a flat universe. The results from WMAP data require $H_{0} r\left(z_{\text {dec }}\right)=3.330_{-0.158}^{+0.193}$ and hence give the allowed range in the shift parameter. The corresponding constraints on MP Cardassian model parameters $(n, q)$ are represented by the dashed line in Fig.2. All models in Figure 2 below the dashed line lie within this allowed range for the shift parameter. We conclude that the Modified Polytropic Cardassian models of Eq.(2) are compatible with current supernova and CMB data. 


\section{Comparison of Models Using Simulated Future Data}

In this section, we will construct simulated type Ia supernova (SN Ia) data for three dark energy models: a Modified Polytropic Cardassian model, a cosmological constant model, and a quintessence model. We then investigate if we can recover the original theory from the simulated data. In particular we want to see if Cardassian cosmology can be differentiated from generic quintessence models or a cosmological constant by analysis of upcoming SN Ia data. We will begin with a modified polytropic Cardassian model of Eq.(2), choose specific values of the parameters $q$ and $n$, and see how many SNe Ia we would expect.

The measured distance modulus for a SN Ia (labeled "l") is

$$
\mu_{0}^{(l)}=\mu_{p}^{(l)}+\epsilon^{(l)}
$$

where $\mu_{p}^{(l)}$ is the theoretical prediction [see Eq.(17)], and $\epsilon^{(l)}$ is the uncertainty in the measurement, including observational errors and intrinsic scatters in the SN Ia absolute magnitudes. In the simulated data set, we take the dispersion in SN Ia peak luminosity to be $\Delta m_{\text {int }}=0.16 \mathrm{mag}$ (this is the rms variance of $\epsilon^{(l)}$ ).

Many (but not all) models of dark energy can be characterized by an equation of state $w_{X}(z)=p_{X}(z) / \rho_{X}(z)$, where $p_{X}(z)$ is the pressure. Most authors have concentrated on constraining the equation of state $w_{X}$ of the dark energy from SN data. However, it was shown by Maor, Brustein, \& Steinhardt (2001); Barger \& Marfatia (2001) that it is extremely hard to constrain $w_{X}$ using SN data. Instead, Wang \& Garnavich (2001) emphasized that it is easier to extract information on the dark energy density $\rho_{X}(z)$, instead of $w_{X}(z)$, from the data. This is because there are multiple integrals relating $w_{X}(z)$ to the luminosity distance $d_{L}(z)$ of SN, which results in a "smearing" that obscures the difference between different $w_{X}(z)$. It is better to use $\rho_{X}(z)$ directly, as it is related to the time derivative of the comoving distance to SN Ia, $r^{\prime}(z)$; hence it is less affected by the smearing effect. The advantage of measuring $\rho_{X}(z)$ over measuring $w_{X}(z)$ was confirmed by Tegmark (2002). In

their work, Wang \& Garnavich (2001) assumed that $\rho_{X}^{\prime}(z)>0$, a condition equivalent to the weak energy condition for those cases in which the ordinary Friedmann equation applies. Here, on the other hand, we make no such assumption. In fact, we will show that it is possible to determine the sign of $\rho_{X}^{\prime}(z)$. 


\subsection{Determining the sign of the time dependence of dark energy density, with prior on $\Omega_{m}$}

We simulate data for three models (see Table 1): (1) a cosmological constant model; (2) a MP Cardassian model with $n=0.2$ and $q=2$, which has $\rho_{X}^{\prime}(z) \leq 0$; (3) a quintessence model with $w_{X}(z)=-1+0.5 z$, which has $\rho_{X}^{\prime}(z) \geq 0$.

Table 1

Dark Energy Models

\begin{tabular}{|l|l|l||}
\hline Model & model parameters & $\rho_{X}^{\prime}(z)$ \\
\hline$\Lambda$ CDM & $\Omega_{m}=0.3, \Omega_{\Lambda}=0.7$ & $\rho_{X}^{\prime}(z)=0$ \\
MP Cardassian model & $\Omega_{m}^{\text {obs }}=0.3, n=0.2, q=2$ & $\rho_{X}^{\prime}(z)<0$ \\
quintessence model & $\Omega_{m}=0.3, w_{q}(z)=-1+0.5 z$ & $\rho_{X}^{\prime}(z) \geq 0$ \\
\hline
\end{tabular}

Note that MP Cardassian models can have either $\rho_{X}^{\prime}(z) \geq 0$ or $\rho_{X}^{\prime}(z)<0$. On the other hand, popular quintessence models in which the quintessence field tracks the matter field have $\rho_{X}^{\prime}(z) \geq 0$ (Barger \& Marfatia 2001). Fig. 3 shows the sign of $\rho_{X}^{\prime}(z)$ in the $(n, q)$ parameter space at fixed $\Omega_{m}=0.3$ for MP Cardassian models [see Eq.(2)] with $\Omega_{m}^{o b s}=0.3$. The arrows indicate the regions in which $\rho_{X}^{\prime}(z) \geq 0$ and $\rho_{X}^{\prime}(z)<0$ respectively. The region in between indicates models with dark energy densities that are not monotonic functions of time.

Note that the weak energy condition requires that the total equation of state $w \geq-1$. In the context of treating Cardassian models as a fluid (Gondolo \& Freese 2002), this is

$$
w=\frac{p_{X}}{\rho_{m}+\rho_{X}}=-\frac{\left[\left(\Omega_{m}^{o b s}\right)^{-q}-1\right](1-n)}{(1+z)^{3(1-n) q}+\left(\Omega_{m}^{o b s}\right)^{-q}-1},
$$

which satisfies $w \geq-1$ for the parameter choices we are interested in: $\Omega_{m}^{o b s}<1, n<1$, and $q>1$. Therefore, all viable MP Cardassian models satisfy the weak energy condition but can have $w_{X}<-1$. Note an effective $w_{X}<-1$ is consistent with recent CMB and large scale structure data (Schuecker et al. 2002; Melchiorri et al. 2002).

Some scalar field dark energy models with $w_{X}<-1$ have been studied previously (Caldwell 1999); models which are stable despite violating variants of the weak energy conditions have been found to be difficult to construct (Carroll, Hoffman, \& Trodden 2003). Our proposal here is a different alternative to the models previously studied. 
We will now assume that the data set is given, either from the simulated data sets described above, or, in the future from $\mathrm{SNAP}^{8}$. We will show that investigation of the data set can reproduce the sign of the time-dependence of the dark energy density, assuming one knows the matter density to an accuracy of $10 \%$.

Given our data set, we now proceed as though we have no information on where it comes from; i.e., we proceed as though we did not know which model it came from. We parametrize the dark energy density in order to allow us to compare it to the data set. We take $\rho_{X}(z)$ to be an arbitrary function. To approximate the function, we parametrize it by its value at $n_{\text {bin }}$ equally spaced redshift values, $z_{i}, i=1,2, \ldots, n_{b i n}, z_{n_{b i n}}=z_{\max }$. The value of $\rho_{X}(z)$ at other redshifts are given by linear interpolation, i.e.,

$$
\begin{aligned}
& \rho_{X}(z)=\left(\frac{z_{i}-z}{z_{i}-z_{i-1}}\right) \rho_{i-1}+\left(\frac{z-z_{i-1}}{z_{i}-z_{i-1}}\right) \rho_{i}, \quad z_{i-1}<z \leq z_{i}, \\
& z_{0}=0, z_{n_{\text {bin }}}=z_{\max } .
\end{aligned}
$$

The values of the dark energy density $\rho_{i}\left(i=1,2, \ldots, n_{\text {bin }}\right)$ are the independent variables to be estimated from data; note that the number of independent variables is $n_{\text {bin }}$. Again, we proceed as though we had absolutely no information on the function $\rho_{X}(z)$, and treat it as a completely arbitrary function.

The complete set of parameters, then, is

$$
\mathbf{s} \equiv\left(\Omega_{m}^{o b s}, \rho_{i}, \text { and } n_{b i n}\right)
$$

where $i=1, \ldots, n_{\text {bin }}$ as described above. Hence our number of parameters is $N=n_{\text {bin }}+2$. We will vary the number of bins $n_{\text {bin }}$ between 1 and 10, and look for the optimal fit to the data. To illustrate, an arbitrary function may become a good approximation to the data for 4 bins whereas it is a miserable fit for 3 bins.

We expand the adaptive iteration method developed in Wang \& Garnavich (2001) and Wang \& Lovelace (2001); unlike what is done in those papers, we do not restrict ourselves to cases where $\rho_{X}^{\prime}(z)>0$.

We can now determine a best fit to the set of parameters $\mathbf{s}$ by using a $\chi^{2}$ statistic, with (Riess et al. 1998)

$$
\chi^{2}(\mathbf{s})=\sum_{l} \frac{\left[\mu_{p}^{(l)}\left(z_{l} \mid \mathbf{s}\right)-\mu_{0}^{(l)}\left(z_{l}\right)\right]^{2}}{\sigma_{l}^{2}},
$$

\footnotetext{
${ }^{8}$ Note that the current SNAP design is sustantially improved than before (Tarle et al. 2002). Here we assume that SNAP will obtain all SNe Ia in its survey fields up to $z=1.7$, similar to a supernova pencil beam survey (Wang 2000a; Wang \& Lovelace 2001).
} 
where $\mu_{p}^{(l)}\left(z_{l} \mid \mathbf{s}\right)$ is the prediction for the distance modulus at redshift $z_{l}$, given the set of parameters s. Here $\sigma_{l}$ is the dispersion of the measured distance modulus due to intrinsic and observational uncertainties in SN Ia peak luminosity.

To reduce the computation time, we can integrate over the Hubble constant $H_{0}$ analytically, and define a modified $\chi^{2}$ statistic, with

$$
\tilde{\chi}^{2} \equiv \chi_{*}^{2}-\frac{C_{1}}{C_{2}}\left(C_{1}+\frac{2}{5} \ln 10\right)-2 \ln h^{*},
$$

where $h^{*}$ is a fiducial value of the dimensionless Hubble constant $h$,

$$
\mu_{p}^{*} \equiv \mu_{p}\left(h=h^{*}\right)=42.384-5 \log h^{*}+5 \log \left[H_{0} r(1+z)\right]
$$

and

$$
\begin{aligned}
\chi_{*}^{2} & \equiv \sum_{l} \frac{1}{\sigma_{l}^{2}}\left(\mu_{p}^{*(l)}-\mu_{0}^{(l)}\right)^{2} \\
C_{1} & \equiv \sum_{l} \frac{1}{\sigma_{l}^{2}}\left(\mu_{p}^{*(l)}-\mu_{0}^{(l)}\right) \\
C_{2} & \equiv \sum_{l} \frac{1}{\sigma_{l}^{2}} .
\end{aligned}
$$

It is straightforward to check that the derivative of $\tilde{\chi}^{2}$ with respect to $h^{*}$ is zero; hence our results are independent of the choice of $h^{*}$. We take $h^{*}=0.65$.

For a given choice of $n_{b i n}$, we can minimize the modified $\chi^{2}$ statistic of Eq.(24) to find the best fit $\Omega_{m}^{o b s}$ and $\rho_{X}(z)$ (parametrized by $\rho_{i}, i=1,2, \ldots, n_{b i n}$ ). We can find one sigma error bars by finding values with $(\Delta \chi)^{2}=1$ from the minimum.

For each model in Table 1, we obtain four sets of best fit parameters. We apply four different constraints to the arbitrary function $\rho_{X}(z)$ in order to discover which one allows a good fit. The four constraints are:

(i) $\rho_{X}(z)=\rho_{X}(0)=$ constant; i.e., a cosmological constant model;

(ii) $\rho_{X}^{\prime}(z) \geq 0$;

(iii) $\rho_{X}^{\prime}(z)<0$;

and (iv) completely unconstrained $\rho_{X}(z)$.

For each of these constraints, we find the best fit parameters.

Figure 4 shows our results: panels (a) and (b) correspond to the MP Cardassian and quintessence models described in Table 1 respectively. For simulated data of Model 1 of Table 1 , a cosmological constant model, we find that $\Omega_{m}^{o b s}$ is estimated correctly to $1 \%$ accuracy, 
regardless of the assumption made about $\rho_{X}^{\prime}(z)$. For Model 2 (MP Cardassian) and Model 3 (quintessence), Fig.4(a) and (b) show the best fit $\Omega_{m}^{o b s}$, under all of the four constraints above, for $n_{b i n}$ values ranging from 1 to 10 . We find that assuming the wrong sign for $\rho_{X}^{\prime}(z)$ leads to an estimated $\Omega_{m}$ that differs from the assumed $\Omega_{m}^{o b s}$ by more than $10 \%$. The different constraints on the sign of $\rho_{X}^{\prime}(z)$ are represented by different point types. The solid horizontal line is our fiducial value of $\Omega_{m}=0.3$ (i.e., we are assuming that that this is the true value of the matter density), and the dot-dashed horizontal lines indicate $10 \%$ error bars about his fiducial value. We are assuming that $\Omega_{m}$ is known to within $10 \%$ from other data sets.

These plots are not intended to emphasize the dependence of $\Omega_{m}^{o b s}$ on $n_{\text {bin }}$. Indeed, as discussed above, the reason that we have found the best fit $\Omega_{m}$ for a variety of $n_{b i n}$ values is simply that the parametrization of the arbitrary function $\rho_{X}(z)$ may be poor for one value of $n_{\text {bin }}$ but excellent for another; we take a given model to be a good one if it lies within the $10 \%$ range on $\Omega_{m}$ for several values of $n_{b i n}$.

Fig.4(a) and (b) show estimated $\Omega_{m}^{o b s}$ as function of $n_{\text {bin }}$ for Model 2 (MP Cardassian model) and Model 3 (a quintessence model). In Fig.4(a), only the $\Omega_{m}^{o b s}$ values estimated assuming that $\rho_{X}^{\prime}(z) \leq 0$ consistently (i.e., for most values of $n_{\text {bin }}$ ) lie within $10 \%$ of the true value of $\Omega_{m}^{o b s}=0.3$. Hence, we have indeed recovered the correct general time-dependence of the model underlying this set of simulated data. In Fig.4(b), only the $\Omega_{m}^{o b s}$ values estimated assuming that $\rho_{X}^{\prime}(z) \geq 0$ deviate by less than $10 \%$ from the true value of $\Omega_{m}^{\text {obs }}=0.3$. Again, we have recovered the correct general time-dependence of the model underlying this set of simulated data. For all three models, we have been able to correctly ascertain the sign of $\rho_{X}^{\prime}(z)$ with this technique.

This indicates that the estimated $\Omega_{m}^{o b s}$ (for a variety of values of $n_{b i n}$ ), together with a $10 \%$ accurate prior on $\Omega_{m}^{o b s}$, can be used to determine the general time-dependence of the dimensionless dark energy density; i.e., the sign of $\rho_{X}^{\prime}(z)$.

\section{2. $\quad$ Estimating dark energy density from data}

In this section we create a large number of Monte Carlo samples to see how well we can reconstruct the entire function $\rho_{X}(z)$. To create these samples, we need first to identify the best-fit model as follows.

For a given data set, we choose the best-fit model with $n_{b i n}$ [the number of parameters used to parametrize the dimensionless dark energy density $\rho_{X}(z)$ ] that satisfies three conditions:

(1) it corresponds to an estimated $\Omega_{m}$ value that deviates less than $10 \%$ from the true value; 
(2) as we decrease $n_{b i n}$ from a large value, say, $n_{b i n}=10$, it minimizes the $\chi^{2}$ per degree of freedom, $\chi_{p d f}^{2}=\chi^{2} /\left(N_{\text {data }}-\nu\right)$, without significantly shifting the estimated value of $\Omega_{m}$ (Wang \& Lovelace 2001). $N_{\text {data }}$ is the number of SNe Ia, and $\nu$ is the number of parameters estimated from data. Long dashed lines in Fig.4 show $\chi_{p d f}^{2}$ as function of $n_{b i n}$ on an arbitrary scale $^{9}$

(3) if $\rho_{X}^{\prime}(z) \neq 0$, then $n_{\text {bin }}>1$.

Now we apply the above conditions to Fig.4(a) and (b). In Fig.4(a), as we decrease $n_{\text {bin }}$ from $n_{b i n}=10$, the significant shifting in the estimated $\Omega_{m}$ occurs at $n_{b i n}=6$, which also has the smallest $\chi_{p d f}^{2}$ for $6 \leq n_{\text {bin }} \leq 10$. We find that for the MP Cardassian model, SNAP data yield an optimal $n_{b i n}=6$. In Fig.4(b), as we decrease $n_{b i n}$ from $n_{b i n}=10$, the significant shifting in the estimated $\Omega_{m}$ occurs at $n_{b i n}=3$, which also has the smallest $\chi_{p d f}^{2}$ for $3 \leq n_{b i n} \leq 10$. Hence for the quintessence model, SNAP data yield an optimal $n_{b i n}=3$.

To derive the error distribution of estimated parameters $\Omega_{m}^{\text {obs }}$ and $\rho_{i}\left(i=1,2, \ldots, n_{b i n}\right.$, see Eq.(21)), we create $10^{4}$ Monte Carlo samples by adding dispersion in peak luminosity of $\Delta m_{\text {int }}=0.16 \mathrm{mag}$ to the distance modulus $\mu_{p}(z)$ [see Eq.(17)] predicted by the bestfit model (i.e., assuming that the best-fit model is the true model). This is equivalent to making $10^{4}$ new "observations", each similar to the original data set (Press et al. 1992). The same analysis used to obtain the best-fit model from the data is performed on each Monte Carlo sample. We use the distribution of the resultant estimates of the parameters $\left(\Omega_{m}^{o b s}\right.$ and $\rho_{i}$ ) to derive the mean and $68.3 \%$ and $99.73 \%$ confidence level intervals of the estimated parameters. Wang \& Lovelace (2001) showed that such a Monte Carlo analysis gives less biased estimates of parameters than a maximum likelihood analysis, i.e., the Monte Carlo mean of estimated parameters deviate less from the true values of the parameters.

Fig. 5 shows the estimated dimensionless dark energy density $\rho_{X}(z)$ for a generalized Cardassian model with $n=0.2$ and $q=2$ from simulated SN data from SNAP assuming that we know $\Omega_{m}$ to $10 \%$ accuracy. The solid line indicates the underlying true model for $\rho_{X}(z)$. The horizontal dashed line near the top of the figure indicates $\rho_{X}(z)=\rho_{X}(0)=$ constant, a cosmological constant model. The horizontal dotted line near the bottom of the figure indicates $\rho_{X}(z)=0$. We impose $\rho_{X}(z) \geq 0$. The estimated $\Omega_{m}$ values (obtained by reconstructing the model from the Monte Carlo samples) are listed at the bottom of the plot with $68.3 \%$ confidence level intervals. Where the actual value of $\Omega_{m}$ for the fake data set was $\Omega_{m}=0.3$, we see that the reproduced $\Omega_{m}$ from our Monte Carlo study is $\Omega_{m}=0.298(-0.023,+0.024)$. Indeed this reproduced value lies within $10 \%$ of the correct $\Omega_{m}$.

\footnotetext{
${ }^{9}$ The scale is adjusted for the curve to fit in the figure.
} 
The error bars of the reproduced estimates of $\rho_{X}(z)$ have been computed using $10^{4}$ Monte Carlo random samples derived from the simulated data. The solid error bars and the dotted error bars indicate the $68.3 \%$ and $99.73 \%$ confidence level intervals respectively. Hence, from Fig. 5, we see that a MP Cardassian model with a set of parameters that fit the current observational data, $\Omega_{m}^{o b s}=0.3, n=0.2, q=2$, can be differentiated from a cosmological constant model at $99.73 \%$ confidence level. We have shown the accuracy with which one can reconstruct the form of the dark energy density. We see that SNAP can indeed differentiate between different models.

\section{Discussion and Conclusion}

We have compared a particular form of Cardassian model, the Modified Polytropic Cardassian model of Eq. (2), with existing data from supernovae and cosmic microwave background measurements. We have found that current data constrain the parameter space of the MP Cardassian model.

We have shown that future type Ia supernova (SN Ia) data from SNAP can differentiate various dark energy models (cosmological constant, quintessence, and generalized Cardassian expansion), assuming that $\Omega_{m}$ is known to $10 \%$ accuracy. We have found the interesting result that the sign of the time dependence of the dark energy density can be determined by SNAP.

Further, we have performed Monte Carlo samples to illustrate how well one can reproduce the form of the dark energy density with SNAP. For example, a MP Cardassian model with a set of parameters that fit the current observational data, $\Omega_{m}^{o b s}=0.3, n=0.2, q=2$, can be differentiated from a cosmological constant model at $99.73 \%$ confidence level.

We wish to remark on another test of generalized Cardassian models. There are two independent motivations for these models: 1) they may arise as a consequence of imbedding our observable universe as a 3-brane in higher dimensions (see, e.g., Chung \& Freese (2000)), and 2) these models may be described in terms of a fluid interpretation, in which the dark energy density may be due to self-interaction of dark matter particles (Gondolo \& Freese 2002). In this second interpretation, we have developed a fully relativistic treatment of the resultant modified Euler's equations, Poisson equations, and energy conservation. Then we analyzed (Gondolo \& Freese 2002) the linear growth of density fluctuations in the fluid interpretation. A more complete study of perturbation growth is in progress. Of particular interest is the study of the Integrated Sachs Wolfe effect in the Cosmic Microwave Background. It is possible that the deficit of power on large angular scales (low order multipoles) 
may be explained in generalized Cardassian models.

We thank the Kavli Institute for Theoretical Physics at the University of California, Santa Barbara, for hospitality. Most of this work was performed in September 2002 when all the authors were at the Institute for Theoretical Physics in Santa Barbara. Subsequent dispersal of all the authors to different parts of the country caused a delay in the publication of this paper.

It is a pleasure for us to thank Arlin Crotts, Greg Tarle, the referee, and especially Josh Frieman for helpful comments. This research was supported in part by the National Science Foundation under Grant No. PHY99-07949, NSF CAREER grant AST-0094335 (Y.W.), the Department of Energy grant at the University of Michigan (K.F. and M.L.), the Michigan Center for Theoretical Physics (K.F. and M.L.). K.F. thanks the Aspen Center for Physics, where part of this research was conducted, for hospitality during her stay.

\section{REFERENCES}

Akerlof, C., et al. Resource Book on Dark Energy; Contributions from the Snowmass 2001 Workshop on the Future of Particle Physics, 2001.

Alcock, C., and Paczynski, B., Nature B281, 358 (1979).

Bond,J.R.; Efstathiou, G.; \& Tegmark, M. 1997, MNRAS, 291, L33

Barger, V., and Marfatia, D., Phys. Lett. B498, 67-73 (2001)

Bean, R., Hansen, S., and Melchiorri, A., astro-ph/0201127

Bennett, C.L., et al. 2003, astro-ph/0302207

Binetruy, P., Deffayet, C., and Langlois, D., Nucl. Phys. B565, 269 (2000).

Caldwell, R., astro-ph/9908168.

Caldwell, R., Dave, R., Steinhardt, P., Phys. Rev. Lett.80 1582 (1998)

Carroll, S.M.; Hoffman, M.; \& Trodden, M. 2003, astro-ph/0301273

Chung, D.J., and Freese, K., Phys. Rev. D61, 023511 (2000)

Davis, M., and Newman, J. 1999, ApJ, 513, L95. 
Deffayet, C., Phys. Lett. B502, 199 (2001)

Evrard, A.E., MNRAS 2921997289.

Freese, K., Adams, F.C., Frieman, J.A., and Mottola, E., Nucl. Phys. B287, 797 (1987).

Freese, K., and Lewis, M., astro-ph/0201229, Phys.Lett. B540, 1 (2002).

Freese, K., Conference Proceeding for Meeting on Sources and Detection of Dark Matter and Dark Energy in the Universe, Marina del Rey, CA (2002).

Frieman, J., Hill, J., Stebbins, A., and Waga, I., Phys. Rev. Lett.75 2077 (1995).

Frieman, J. A. 1997, Comments Astrophys., 18, 323

Gondolo, P., and Freese, K., hep-ph/0209322, hep-ph/0211397.

Hannestad, S., and Mortsell, E., astro-ph/0205096

Holz, D.E. \& Wald, R.M. 1998, Phys. Rev. D, 58, 063501

Huey, G., Wang, L., Dave, R., Caldwell, R., and Steinhardt, P., Phys. Rev. D59 063005 (1999)

Hui, L., Stebbins, A., and Burles, S. 1999, ApJ, 511, L5.

Huterer, D., and Turner, M., Phys. Rev. D64, 123527 (2001);

Krauss, L.M., and Chaboyer, B., astro-ph/0111597

Maor, I., Brustein, R., Steinhardt, P.J., Phys. Rev. Lett., 86, 6 (2001); Erratum-ibid. 87 (2001) 049901

McDonald, P., and Miralda-Escude, J. 1999, ApJ, 518, 24.

Melchiorri, A.; Mersini, L.; Ödman, C.J.; \& Trodden, M. 2002, astro-ph/0211522

Metcalf, R. B., \& Silk, J. 1999, ApJ, 519, L1

Multamaki, T.; Gaztanaga, E.; \& Manera, M. 2003, astro-ph/0303526

Munshi, D., and Wang, Y. 2003, ApJ, 583, 566

Netterfield, C.B., et al, astro-ph/0104460

Ödman, C.J.; Melchiorri, A.; Hobson, M.P.; \& Lasenby, A.N. 2002, astro-ph/0207286 
Peebles, P. J. E.; Ratra, B. 1988, ApJ, 325L, 17

Peebles, P.J.E., "Principles of Physical Cosmology" (Princeton University Press, Princeton, NJ, 1993).

Perlmutter, S., et al. [Supernova Cosmology Project Collaboration], Astrophys. J. 517, 565 (1999) [arXiv:astro-ph/9812133].

Phillips, M.M., ApJ, 413, L105 (1993)

Press, W. H., Teukolsky, S. A., Vetterling, W. T., Flannery, B.P., "Numerical Recipes in Fortran 77 (The Art of Scientific Computing)" (Cambridge University Press, 2nd ed., 1992)

Pryke, C. ,et al 2002, ApJ, 568, 46

Riess, A.G., Press, W.H., and Kirshner, R.P., ApJ, 438, L17 (1995)

Riess, A. G., et al. [Supernova Search Team Collaboration], Astron. J. 116, 1009 (1998) [arXiv:astro-ph/9805201].

Schuecker, P. et al. 2002, astro-ph/0211480

Sen, S., and Sen, A. A., astro-ph/0211634.

Spergel, D.N., et al. 2003, astro-ph/0302209

Tarle, G., (for the SNAP Collaboration), astro-ph/0210041

Tegmark, M., Phys. Rev. D66, 103507 (2002)

Wambsganss, J., Cen, R., Xu, G., \& Ostriker, J.P. 1997, ApJ, 475, L81

Wang, L., and Steinhardt, P. 1998, ApJ, 508, 483.

Wang, Y. 1999, ApJ, 525, 651

Wang, Y. 2000a, ApJ, 531, 676

Wang, Y. 2000b, ApJ, 536, 531

Wang, Y., and Garnavich, P. 2001, ApJ, 552, 445

Wang, Y., and Lovelace, G. 2001, ApJ, 562, L115 
Wang, Y., Holz, D.E., and Munshi, D. 2002, ApJ, 572, L15 (2002)

Weinberg, S., "Gravitation and Cosmology" (John Wiley \& Sons, New York, 1972)

White, S.D.M., Navarro, J.F., Evrard, A.E., and Frenk, C.S., Nature B366, 429 (1993).

Zhu, Z., \& Fujimoto, M. 2002, ApJ, 581, 1

Zhu, Z., \& Fujimoto, M. 2003, ApJ submitted 
Fig. 1. - Examples of MP Cardassian models [see Eq.(2)] that satisfy current observational constraints from type Ia supernovae (SNe Ia) data. Three MP Cardassian models are shown, all with $\Omega_{m}^{o b s}=0.3: n=0.2, q=1$ (solid curve); $n=0.2, q=2$ (short-dashed curve); and $n=0.2, q=3$ (long-dashed curve). Note that the solid curve is equivalent to a quintessence model with $w_{q}=-0.8$. The dot-dashed curve is a quintessence model with $w_{q}=-1+0.5 z$. The dotted curves show several familiar cosmological models for comparison (from top to bottom): $\left(\Omega_{m}, \Omega_{\Lambda}\right)=(0.3,0.7) ;(0.3,0)$; and $(1,0)$.

Fig. 2.- The parameter space of $(n, q)$ showing constraints from SNe Ia and CMB at fixed $\Omega_{m}=0.3$. All models below the dashed line are in agreement with the shift parameter $\mathcal{R}$ as measured by WMAP. All models between the solid lines are in agreement with the Type Ia SN data.

Fig. 3. - The parameter space of $(\mathrm{n}, \mathrm{q})$ for MP Cardassian models [see Eq.(2)] with $\Omega_{m}^{\text {obs }}=$ 0.3. The arrows indicate the regions in which $\rho_{X}^{\prime}(z) \geq 0$ and $\rho_{X}^{\prime}(z)<0$ respectively. The region in between indicates models with dark energy densities that are not monotonic functions of time.

Fig. 4. - This figure shows that we can indeed determine the sign of the dependence of the dark energy density $\rho_{X}^{\prime}(z)$; i.e., we can determine if it is increasing, decreasing, or constant in time. The axes show estimated $\Omega_{m}^{e s t}$ as function of $n_{b i n}$ from the simulated data for SNAP for (a) a MP Cardassian model with $n=0.2$ and $q=2$ so that $\rho_{X}^{\prime}(z)<0$ and (b) a quintessence model with $w_{X}(z)=-1+0.5 z$ so that $\rho_{X}^{\prime}(z)>0$. The horizontal dot-dashed lines correspond to a $10 \%$ uncertainty on $\Omega_{m}^{o b s}=0.3 \pm 0.03$. The different curves show the results obtained assuming a variety of constraints on the time dependence $\rho_{X}^{\prime}(z)$ as labeled. The long-dashed curve shows $\chi_{p d f}^{2}$ on an arbitrary scale. By requiring the results to lie within the dot-dashed lines, we recover the sign of the time-dependence of $\rho_{X}(z)$.

Fig. 5.- Estimated dimensionless dark energy density $\rho_{X}(z)$ for simulated SN data from SNAP, assuming that we know $\Omega_{m}$ to $\sim 10 \%$ accuracy [and the correct general time dependence of the dark energy density, see discussion in Section 3.A]. The error bars of the estimated $\rho_{X}(z)$ have been computed from $10^{4}$ Monte Carlo random samples derived from the simulated data. The solid error bars and the dotted error bars indicate the $68.3 \%$ and $99.73 \%$ confidence level intervals respectively. The reproduced estimates of $\Omega_{m}$ values are listed at the bottom of the plot with $68.3 \%$ confidence level intervals. 


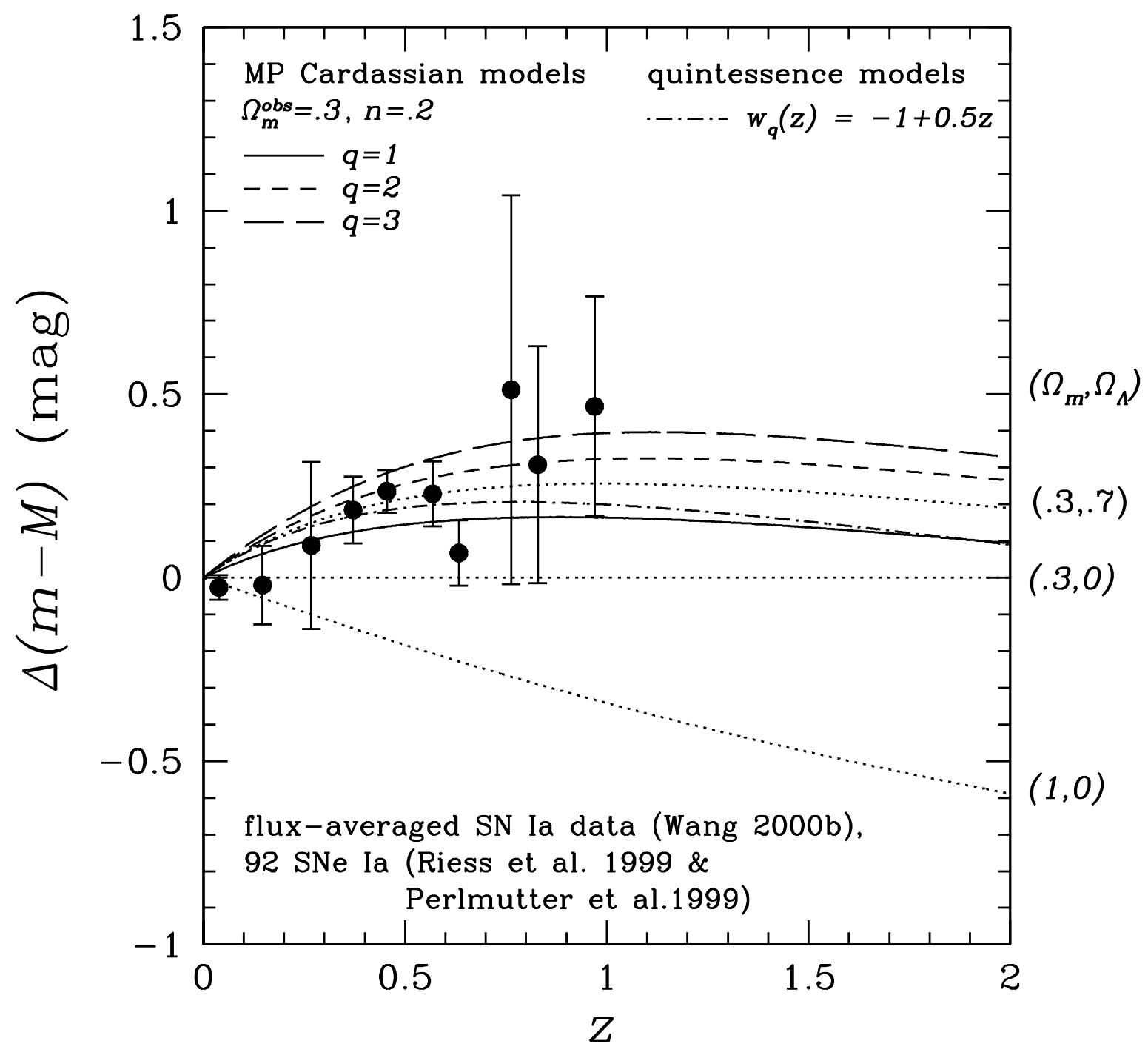

Fig. 1.- Examples of MP Cardassian models [see Eq.(2)] that satisfy current observational constraints from type Ia supernovae (SNe Ia) data. Three MP Cardassian models are shown, all with $\Omega_{m}^{o b s}=0.3: n=0.2, q=1$ (solid curve); $n=0.2, q=2$ (short-dashed curve); and $n=0.2, q=3$ (long-dashed curve). Note that the solid curve is equivalent to a quintessence model with $w_{q}=-0.8$. The dot-dashed curve is a quintessence model with $w_{q}=-1+0.5 z$. The dotted curves show several familiar cosmological models for comparison (from top to bottom): $\left(\Omega_{m}, \Omega_{\Lambda}\right)=(0.3,0.7) ;(0.3,0)$; and $(1,0)$. 


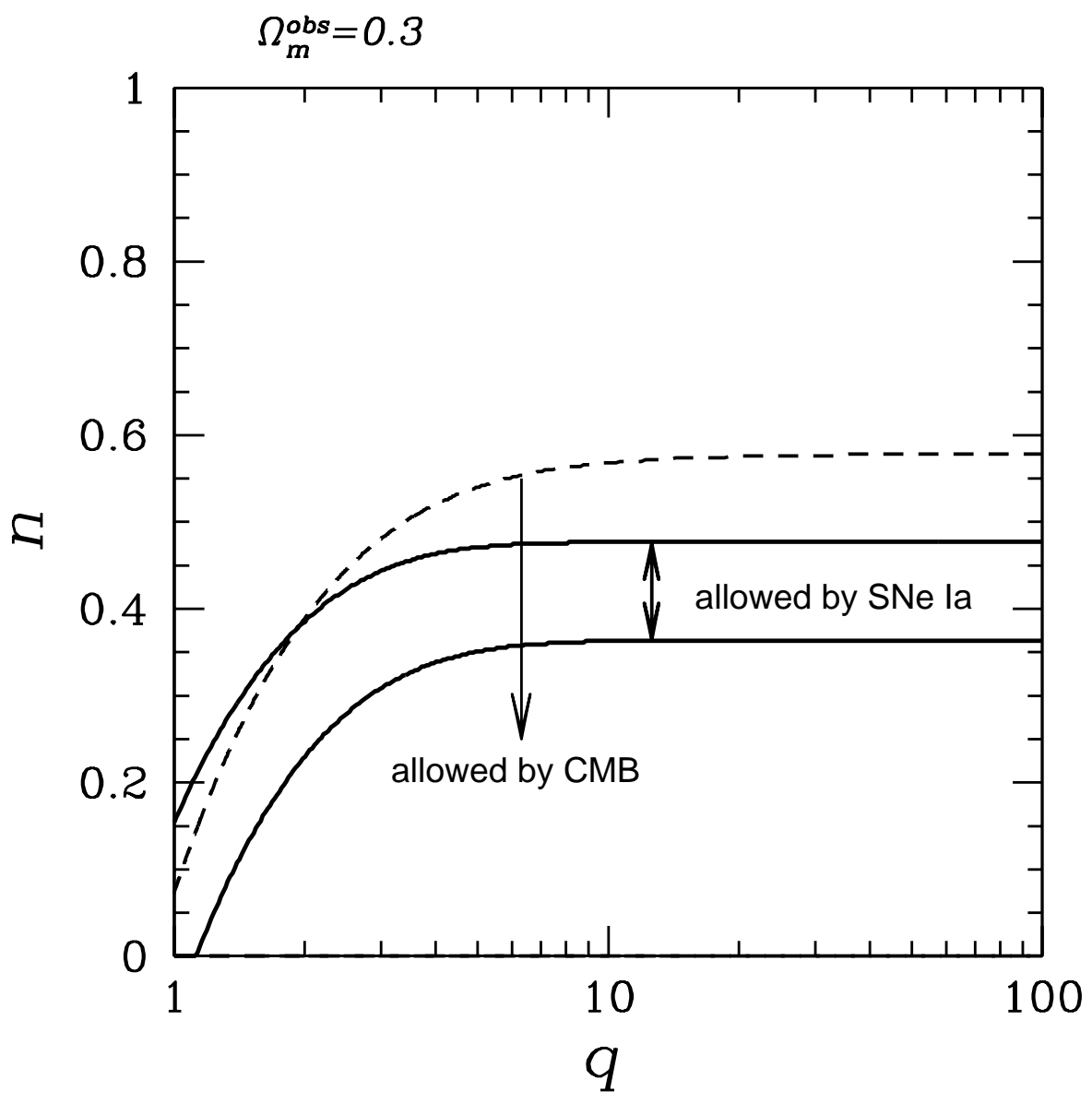

Fig. 2.- The parameter space of $(n, q)$ showing constraints from SNe Ia and CMB at fixed $\Omega_{m}=0.3$. All models below the dashed line are in agreement with the shift parameter $\mathcal{R}$ as measured by WMAP. All models between the solid lines are in agreement with the Type Ia SN data. 


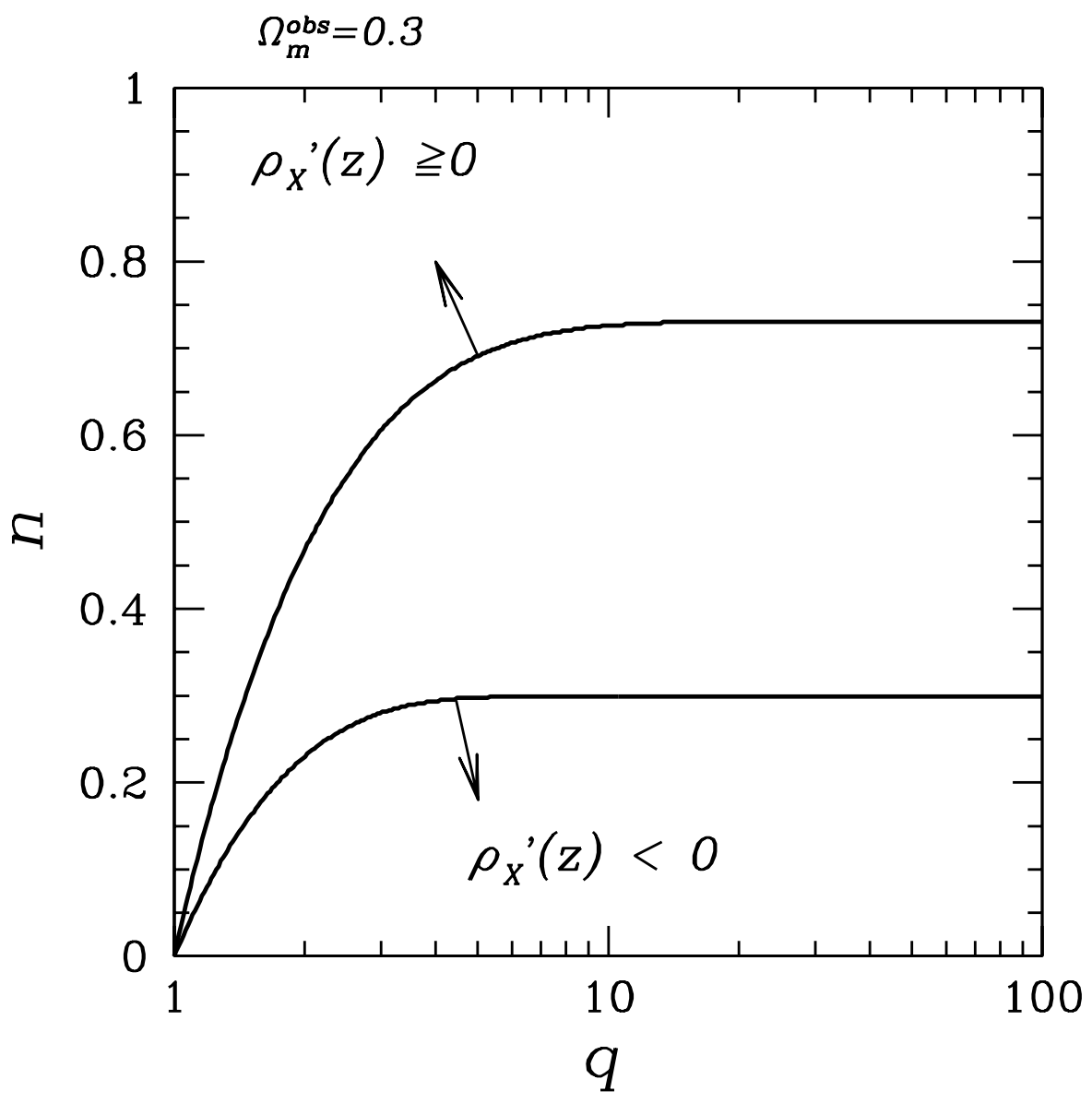

Fig. 3. - The parameter space of (n,q) for MP Cardassian models [see Eq.(2)] with $\Omega_{m}^{\text {obs }}=$ 0.3. The arrows indicate the regions in which $\rho_{X}^{\prime}(z) \geq 0$ and $\rho_{X}^{\prime}(z)<0$ respectively. The region in between indicates models with dark energy densities that are not monotonic functions of time. 

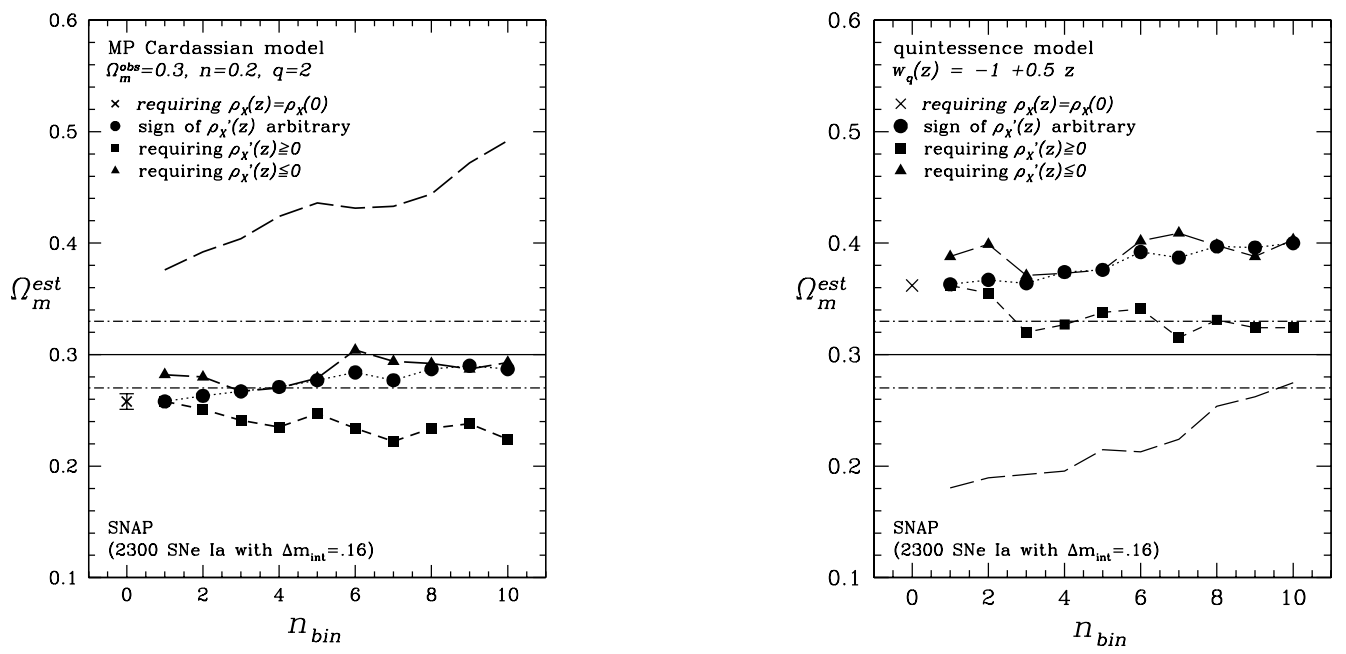

Fig. 4. - This figure shows that we can indeed determine the sign of the dependence of the dark energy density $\rho_{X}^{\prime}(z)$; i.e., we can determine if it is increasing, decreasing, or constant in time. The axes show estimated $\Omega_{m}^{e s t}$ as function of $n_{b i n}$ from the simulated data for SNAP for (a) a MP Cardassian model with $n=0.2$ and $q=2$ so that $\rho_{X}^{\prime}(z)<0$ and (b) a quintessence model with $w_{X}(z)=-1+0.5 z$ so that $\rho_{X}^{\prime}(z)>0$. The horizontal dot-dashed lines correspond to a $10 \%$ uncertainty on $\Omega_{m}^{o b s}=0.3 \pm 0.03$. The different curves show the results obtained assuming a variety of constraints on the time dependence $\rho_{X}^{\prime}(z)$ as labeled. The long-dashed curve shows $\chi_{p d f}^{2}$ on an arbitrary scale. By requiring the results to lie within the dot-dashed lines, we recover the sign of the time-dependence of $\rho_{X}(z)$.

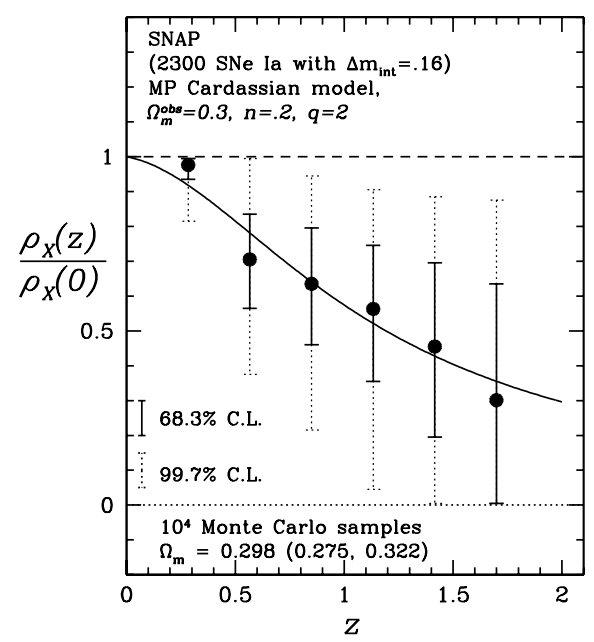


Fig. 5.- Estimated dimensionless dark energy density $\rho_{X}(z)$ for simulated SN data from SNAP, assuming that we know $\Omega_{m}$ to $\sim 10 \%$ accuracy [and the correct general time dependence of the dark energy density, see discussion in Section 3.A]. The error bars of the estimated $\rho_{X}(z)$ have been computed from $10^{4}$ Monte Carlo random samples derived from the simulated data. The solid error bars and the dotted error bars indicate the $68.3 \%$ and $99.73 \%$ confidence level intervals respectively. The reproduced estimates of $\Omega_{m}$ values are listed at the bottom of the plot with $68.3 \%$ confidence level intervals. 